\title{
Do Brain Networks Correlate with Intelligence?
}

\author{
Tianzi Jiang \\ LIAMA Center for Computational Medicine \\ National Laboratory of Pattern Recognition \\ Institute of Automation, Chinese Academy of Sciences \\ Beijing 100080, P.R. China \\ jiangtz@nlpr.ia.ac.cn
}

Intuitively, higher intelligence might be assumed to correspond to more efficient information transfer in the brain, but no direct evidence has been reported from the perspective of brain networks. In this lecture, we first give a brief introduction about the basic concepts of brain networks from different scales and classified ways. In the second part, we present the advance on how functional brain networks correlate with intelligence. We focus on the evidence obtained with functional magnetic resonance imaging (fMRI) in the rest state. In the third part of this lecture, we will discuss how individual differences in intelligence are associated with brain structural organization, and in particular that higher scores on intelligence tests are related to greater global efficiency of the brain anatomical network. We focus on the evidence obtained with diffusion tensor imaging (DTI), a type of magnetic resonance imaging. In the fourth part, we discuss the genetic basis of intelligence-related brain networks. We try to address the issue on how intelligence-related genes influence intelligence-related neuronal systems. The evidence based on fMRI and DTI are presented. Finally, the future directions in this field will be presented. 